\title{
GRBS AND LOBSTER EYE X-RAY TELESCOPES
}

\author{
R. Hudec ${ }^{1,2}$, L. Pina ${ }^{3}$, V. Marsikova ${ }^{4}$ and A. Inneman ${ }^{4}$
}

\begin{abstract}
A large majority of GRBs exhibit X-ray emission. In addition, a dedicated separate group of GRB, the XRFs, exists which emission dominates in the $\mathrm{X}$-ray spectral range. And the third group of GRB related objects (yet hypothetical) are the group of off-axis observed GRBs (orphan afterglows). These facts justify the consideration of an independent experiment for monitoring, detection and analyses of GRBs and others fast X-ray transients in X-rays. We will present and discuss such experiment based on wide-field X-ray telescopes of Lobster Eye type. We show that the wide field and fine sensitivity of Lobster Eye X-ray All-Sky Monitor make such instruments important tools in study of GRBs.
\end{abstract}

\section{Introduction}

The X-ray sky is highly variable, rich in variable and transient sources of both galactic as well as extragalactic origin. Among physically most important transient sources, the detection of Gamma Ray Bursts (GRBs) in X-rays confirms the feasibility of monitoring, detecting and study of these phenomena by their X-ray emission (either prompt or afterglow, e.g. Amati et al. 2004 \& Fontera et al. 2004). For classical GRBs, the X-ray afterglows are detected in $~ 90 \%$ of the cases (De Pasquale et al. 2003). Moreover, there are X-ray rich GRBs, (hypothetical) orphan GRBs (detectable in X-rays but not in gamma-rays due to different beaming angle) and XRFs which can be detected and studied in X-rays. However, since these events cannot be predicted, and are relatively rare, very wide-field instruments are required. They must achieve high sensitivities and provide precise

\footnotetext{
1 Astronomical Institute, Academy of Sciences of the Czech Republic, 25165 Ondřejov, Czech Republic

${ }^{2}$ Czech Technical University in Prague, Faculty of Electrical Engineering, Prague, Czech Republic

${ }^{3}$ Czech Technical University in Prague, Faculty of Nuclear Science, Prague, Czech Republic

${ }^{4}$ Rigaku Innovative Techniologies Europe, Prague, Czech Republic
} 

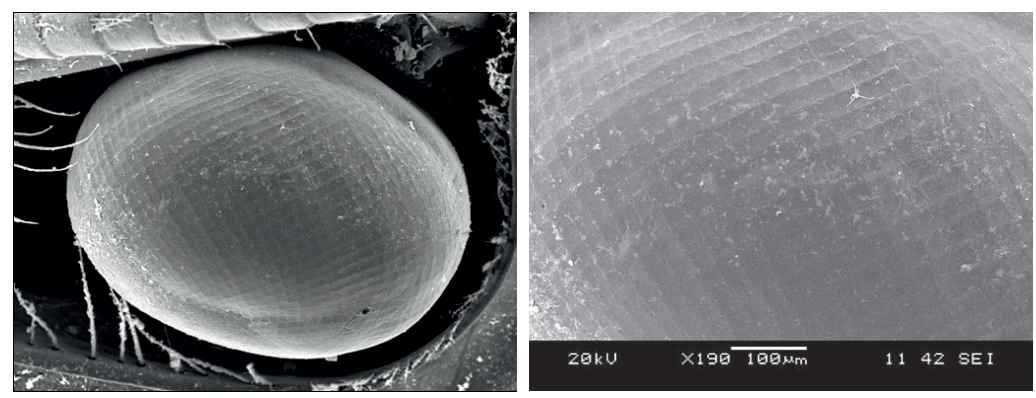

Fig. 1. The crayfish eye under optical miscroscope. On the picture right the surface square cells (covered by protecting membrane) are clearly visible.

localizations in order to effectively study the objects. Novel wide field X-ray telescopes with imaging optics are expected to represent an important tool in future space astronomy projects in general, especially those for deep monitoring and surveys in X-rays over a wide energy range. The Lobster-Eye (LE) wide field X-ray optics has been suggested by Schmidt (Schmidt 1975, orthogonal stacks of reflectors) and by Angel (Angel 1979, array of square cells). Up to 180 deg FOV may be achieved with these devices. This X-ray optics offers an opportunity to achieve very wide fields of view (FOV, 1000 square degrees and more) while the widely used classical Wolter grazing incidence mirrors are limited to roughly 1 deg FOV (Priedhorsky et al. 1996; Inneman et al. 2000).

\section{Lobster eye X-ray telescopes}

The LE telescopes in Schmidt arrangements are based on perpendicular arrays of double-sided X-ray reflecting flats. In the first prototypes developed and tested, double-sided reflecting flats produced by epoxy sandwich technology as well as gold coated glass foils have been used (Inneman et al. 1999). More recently, micro Schmidt lobster eye arrays with foils thickness as low as 30 microns have been developed and tested in order to confirm the capability of these systems to achieve fine angular resolutions of order of a few arcmin (Fig. 1). The thin foils are separated by 70 microns gaps in these prototypes. On the other hand, large lobster eye systems with Schmidt geometry have been designed and constructed, achieving dimensions up to $300 \times 300 \times 600 \mathrm{~mm}$ (Fig. 1). Their optical and X-ray optical tests (Fig. 2) have confirmed the expected performance according to calculations (computer ray-tracing). The calculations and the measurement results indicate that the lobster eye telescope based on multi array of modules with thin and closely spaced glass foils (analogous to those already assembled and tested) can meet the requirements e.g. of the ESA ISS Lobster mission (including the angular resolution and with better transmission) and can hence represent an alternative to the recently suggested MCP technique (Fraser et al. 2002). 


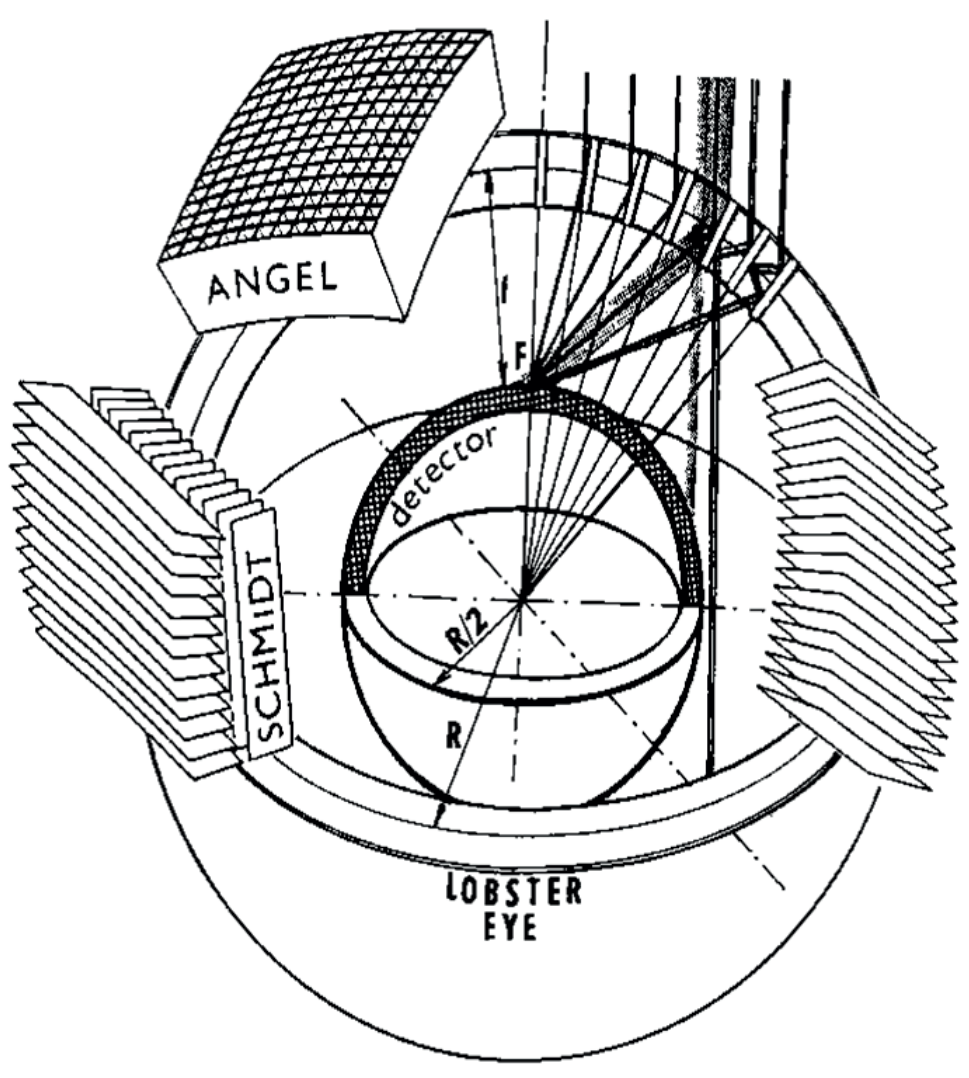

Fig. 2. The two arrangements of the LE X-ray optics.

The Angel LE systems consist of numerous square cells of very small size (about $1 \times 1 \mathrm{~mm}$ or less at lengths of order of tens of $\mathrm{mm}$, i.e. with the length/size ratio of 30 and more) are to be produced. Test modules with LE Angel cells have been successfully produced. The linear test module has 47 cells $2.5 \times 2.5 \mathrm{~mm}$, $120 \mathrm{~mm}$ long (i.e. length/size ratio of almost 50), surface microroughness $0.8 \mathrm{~nm}$, $f=1300 \mathrm{~mm}$. Another test module is represented by a L-shaped array of $2 \times 18=36$ cells of analogous dimension. The surface microroughness of the replicated reflecting surfaces is better than $1 \mathrm{~nm}$.

\section{Astrophysics with LE X-ray monitor}

Deep (limiting flux of $10^{-12} \mathrm{erg} \mathrm{cm}^{-2} \mathrm{~s}^{-1}$ can be easily achieved for daily scanning observation) X-ray sky monitoring with large FOVs (e.g. FOV of $6 \times 180 \mathrm{deg}$ can be easily assembled on the space vehicles) is expected to contribute significantly to various fields of modern astrophysics (2009). A few most important examples are listed below. 


\begin{tabular}{|c|c|c|c|c|c|c|c|c|c|}
\hline \multirow[t]{2}{*}{ MODULE } & size & \begin{tabular}{|c|} 
plate \\
thickness
\end{tabular} & distance & length & $\begin{array}{c}\text { eff. } \\
\text { angle }\end{array}$ & \begin{tabular}{|l|} 
focal \\
length
\end{tabular} & resolution & $\begin{array}{c}\text { field } \\
\text { of view }\end{array}$ & energy \\
\hline & $\mathrm{d}(\mathrm{mm})$ & $\mathrm{t}(\mathrm{mm})$ & $a(m m)$ & $\mathrm{I}(\mathrm{mm})$ & $a / l$ & $f(m m)$ & $r(\operatorname{arcmin})$ & $\left({ }^{\circ}\right)$ & $(\mathrm{keV})$ \\
\hline macro & 300 & 0.75 & 10.80 & 300 & 0.036 & 6000 & 7 & 16 & 3 \\
\hline middle & 80 & 0.3 & 2 & 80 & 0.025 & 400 & 20 & 12 & 2 \\
\hline $\operatorname{mini} 1$ & 24 & 0.1 & 0.3 & 30 & 0.01 & 900 & 2 & 5 & 5 \\
\hline $\min 12$ & 24 & 0.1 & 0.3 & 30 & 0.01 & 250 & 6 & 5 & 5 \\
\hline micro & 3 & 0.03 & 0.07 & 14 & 0.005 & 80 & 4 & 3 & 10 \\
\hline
\end{tabular}

Fig. 3. The LE laboratory samples assembed and tested in the Czech Republic.

(1) Gamma Ray Bursts (GRBs). Detection rates of nearly 20 GRBs/year can be obtained for the prompt X-ray emission of GRBs, taking into account the expected GRB rate 300/year. (2) X-ray flashes. Detection rates of nearly 8 X-ray flashes/year are expected, assuming XRF rate of 100/year. (3) X-ray binaries. Because of their high variability in X-rays they will be one of major targets in LE observations. LE will be able to observe their short-time outbursts by longterm extended monitoring. Almost all galactic XRB are expected to be within the detection limits. (4) Stars. Because of the low X-ray luminosity of ordinary stars, only nearby stars are expected to be observable. We estimate the lower limit of ordinary stars observable by the LE telescope as 600 . The sampling rate of LE observations will be sufficient enough to observe sudden X-ray flux increases during flares while still having the capability of monitoring the variability on time scales of years. (5) Supernovae. The LE telescope should be able to detect the theoretically predicted thermal flash lasting for $\sim 1000 \mathrm{sec}$ for the first time. Together with the optical SNe detection rate and estimates of the LE FOV we estimate the total number of SNe thermal flashes observed by the LE experiment to $~ 10 /$ year. More details on the advantages of LE X-ray telescopes in scientific analysis of SNe are given in Sveda et al. 2005. (6) AGNs. Active Galactic Nuclei will surely be one of the key targets of the LE experiment. LE will be able to monitor the behavior of the large $(\sim 1000)$ sample of AGNs providing long-term observational data with good time sampling (hours). (7) X-ray transients. The LE experiment will be ideal to observe X-ray transients of various nature due to its ability to observe the whole sky several times a day for a long time with a limiting flux of about $10^{-12} \mathrm{erg}^{-2} \mathrm{~cm}^{-1}$. More and fainter X-ray transients are expected to be detected by the LE sky monitor enabling the detailed study of these phenomena. (8) Cataclysmic Variables. Cataclysmic Variables (CVs) are very active galactic objects, often showing violent long-term activity in both the optical and X-ray passband (outbursts, high/low state transitions, nova explosions) as well as rapid transitions between the states of activity. Search for the relation of the optical and X-ray activity is very important - monitoring of a large number of CVs is necessary to catch them in various states of activity.

\section{Conclusions}

The various prototypes of both Schmidt as well as Angel arrangements have been produced and tested successfully, demonstrating the possibility to construct these 
lenses by innovative but feasible technologies. Both very small Schmidt lenses $(3 \times 3 \mathrm{~mm})$ as well as large lenses $(300 \times 300 \mathrm{~mm})$ were developed, constructed, and tested $(1999,2000)$. Promising results were obtained in studies of LE X-ray monitors for small satellites and related tests $(2009,2009,2011)$. This makes the proposals for space projects with very wide field lobster eye optics possible for the first time. The LE All Sky Monitor is capable to detect around 20 GRBs and 8 XRFs yearly and this will surely significantly contribute to the related science. Another LE application may be the investigation of X-ray emmission from atmospheric triggers such as Terrestrial Gamma-Ray Flashes (TGF).

This study and related scientific considerations were partly supported by the grants 102/09/0997 and 13-33324S Lobster Eye X-Ray Monitor provided by the Grant Agency of the Czech Republic. The microscopic images of crayfish eye were kindly provided by Petr Jan Juračka and Adam Petrušek, Charles University, Faculty of Natural Sciences in Prague.

\section{References}

Amati, L., Frontera, F., in't Zand, J.J.M., et al., 2004, A\&A, 426, 415

Angel, J.R.P., 1979, ApJ, 364, 233

De Pasquale, M., Piro, L., Perna, R., et al., 2003, ApJ, 592, 1018

Fraser, G.W., Brunton, A.N., Bannister, N.P., et al., 2002, Proc. SPIE, 4497, 115

Frontera, F., Amati, L., in 't Zand, J.J.M., et al., 2004, ApJ, 616, 1078

Gorenstein, P., 1998, Proc. SPIE, 3444, 382

Inneman, A., Hudec, R., \& Pina, L., 2000, Proc. SPIE, 4138, 94

Inneman, A., Hudec, R., Pina, L., et al., 1999, Proc. SPIE, 3766, 72

Priedhorsky, W.C., Peele, A.G., \& Nugent, K.A., 1996, MNRAS, 279, 733

Schmidt, W.K.H., 1975, Nucl. Instr. Meth., 127, 285

Sveda, L., Hudec, R., Pina, L., et al., 2005, in Cosmic Explosions, Springer Proceedings in Physics, Vol. 99, ed. J.M. Marcaide \& K.W. Weiler, 197

Sveda, L., Hudec, R., Pina, L., et al., 2009, in EUV and X-Ray Optics: Synergy between Laboratory and Space, ed. R. Hudec \& L. Pina, Proceedings of the SPIE, Vol. 7360, p. $73600 \mathrm{~F}, 10$

Tichy, V., Sveda, L., Marsik, J., et al., 2009a, Baltic Astron., 18, 369

Tichy, V., Hromcik, M., Hudec, R., et al., 2009b, Baltic Astron., 18, 362

Tichy, V., Barbera, M., Collura, A., et al., 2011, Nucl. Instr. Meth. Phys. Res. A, 633, id. 169 
